\title{
Dimensiones de la personalidad y procrastinación académica en estudiantes de cursos online de una universidad peruana
}

\section{Personality dimensions and academic procrastination in students of online courses at a Peruvian university}

\author{
José Charaña Mamani, ${ }^{1 \mathrm{a}}$ y Juanito Vílchez Julon² \\ Universidad Peruana Unión, Lima, Perú ${ }^{12}$ \\ Orcid ID: https://orcid.org/0000-0002-4638-6450 \\ Orcid ID: https://orcid.org/0000-0003-4760-6547
}

Recibido: 01 de diciembre de 2020

Aceptado: 26 de marzo de 2021

\begin{abstract}
Resumen
La presente investigación tuvo como objetivo determinar la relación entre las dimensiones de la personalidad y procrastinación académica, además de describir los niveles de procrastinación según edad y sexo en estudiantes de cursos online de una universidad peruana de la ciudad de Tarapoto, 2020. El tipo de investigación fue de diseño no experimental transversal de tipo correlacional, de muestreo no probabilístico por conveniencia, la muestra estuvo conformada por 240 estudiantes universitarios cuyas edades oscilaban entre 16 a 40 años (66.3\% mujeres y $33.7 \%$ varones; edad media $=20.66)$. Los instrumentos utilizados fue la escala de procrastinación académica (EPA) y el Big Five Inventory-15P (BFI-15P). Los resultados revelaron una correlación inversa significativa entre las dimensiones de la personalidad y procrastinación académica; responsabilidad (rho $=-0.590 ; \mathrm{p}<.001)$, amabilidad (rho= -0.457 ; $\mathrm{p}<.000$ ), extraversión (rho= -0.226; $\mathrm{p}<.000$ ) y apertura (rho $=-355 ; \mathrm{p}<.000$ ); quiere decir que a mayor responsabilidad, amabilidad, extraversión y apertura menor será la procrastinación académica o viceversa. Caso contrario sucede con la dimensión neuroticismo que presenta una significativa correlación directa $(r h o=0.166 ; \mathrm{p}<.000)$, de modo que a mayor neuroticismo, mayor procrastinación académica.
\end{abstract}

Palabras clave: Procrastinación académica; autorregulación; estudiantes universitarios; dimensiones de personalidad.

\begin{abstract}
The present research aimed to determine the relationship between the dimensions of personality and academic procrastination, in addition to describing the levels of procrastination according to age and sex in students of online courses at a Peruvian university in the city of Tarapoto, 2020. The type of investigation was of non-experimental transversal design of correlational type, of non-probability sampling for convenience, the sample was confirmed by 240 university
\end{abstract}


students whose ages oscillated between 16 and 40 years (66.3\% females and 33.7\% males; mean age $=20.66)$. The instruments used were the academic procrastination scale (EPA) and the Big Five Inventory-15P (BFI-15P). The results revealed a significant inverse correlation between the dimensions of personality and academic procrastination; responsibility (rho= 0.590; $\mathrm{p}<.001$ ), kindness (rho $=-0.457 ; \mathrm{p}<.000$ ), extraversion (rho $=-0.226 ; \mathrm{p}<.000$ ) and openness ( $\mathrm{rho}=-355 ; \mathrm{p}<.000$ ); it means that the greater the responsibility, kindness, extraversion and openness the less academic procrastination will be or vice versa. The opposite happens with the neuroticism dimension, which presents a significant direct correlation (rho= $0.166 ; \mathrm{p}<.000$ ), so that the greater the neuroticism, the greater the academic procrastination.

Keywords: Academic procrastination; self-regulation; University students; personality factors.

\section{Introducción}

La procrastinación es comprendida como el aplazamiento voluntario de compromisos, siendo consciente de las consecuencias negativas (Ayala, 2020). Esta definición general tiene aplicaciones específicas en actividades con un entorno escolar, llamándose procrastinación académica (PA), siendo definida como la acción de retrasar voluntariamente las tareas, llevándolo a consecuencias de malestar psicológico y problemas académicos (Ferreyra et al., 2017).

Un estudio aplicado a ocho países de habla inglesa (USA, Australia, Canadá, India, Irlanda, New Zelanda, Filipinas y Reino Unido) con una muestra de 16 mil 413 participantes indican que un $75 \%$ de estudiantes mantienen conductas de dilación académica y aproximadamente un 15 a un 20\% de adultos postergan problemáticamente (Steel y Ferrari, 2012). Mientras que en el Perú las investigaciones presentadas en distintos artículos varían en un $50 \%$ - 70\% de universitarios que recurren usualmente a conductas procrastinantes, y cerca del 20\% - 35\% evidencian rasgos permanentes con consecuencias problemáticas (Castro y Mahamud, 2017, Quiroga, 2020, Valverde 2020).

Según autores (Díaz-Morales, 2018; Domínguez et al., 2019), la PA tiene dos componentes fundamentales: (a) la autorregulación académica, que es la capacidad activa y constructiva para supervisar y controlar recursos potenciales como la cognición, motivación y el comportamiento para lograr el fin deseado y (b) la postergación de actividades, que son acciones llevadas a cabo para prolongar la realización de la tarea en cuestión, debido a un mal manejo del tiempo y de ejecutar tareas sin ningún orden de prioridad.

Referente al desempeño académico la PA trae consigo consecuencias como una baja autoeficacia en los trabajos, conducta disruptiva, pérdida de motivación hacia tareas futuras, deserción escolar o permanencia más allá del tiempo esperado para terminar sus estudios 
(Cerqueira et al., 2016, Ayala, 2020). En lo emocional, según diversos autores (Bastidas, 2017; Maldonado y Zenteno, 2018; Matalinares et al., 2017), la PA puede contribuir al desarrollo de alteraciones emocionales asociadas a la baja autoestima, vergüenza, culpa, frustración, hostilidad, ansiedad, estrés, fatiga, culpa, miedo al fracaso, depresión, desesperación, inflexibilidad psicológica y depresión. Además de disminuir de la capacidad cognitivas como la planificación, previsión, atención y concentración (Schutte y del Pozo de Bolger, 2020).

Con todo esto explicar el origen y el sustento de esta conducta puede ser complicado y profundo, existen distintos puntos de vista que la explican: Desde una perspectiva psicodinámica la procrastinación estaría vinculada con el concepto de evitación de tareas específicas que es provocada por la angustia, siendo esta misma una alerta para el yo, al señalarle el material inconscientemente reprimido que trata de emerger de modo amenazante, una vez que la angustia es detectada, el yo emplea una variedad de mecanismos de defensa que permitan en algunos casos evitar la tarea (Atalaya y García, 2019).

De otro modo, la perspectiva conductual refiere que la conducta se mantiene cuando es reforzada, es decir las conductas persisten por sus consecuencias (Ayala et al., 2020). Es por ese motivo que las personas procrastinan porque sus conductas han sido reforzadas y han tenido éxito por factores del ambiente (Diaz-Morales, 2018). Mientras la visión cognitiva-conductual Ellis y Knaus, aducen que la procrastinación se debe a que la persona se propone metas altas e irreales que finalmente termina en fracaso. Es entonces que al intentar calmar esas emociones demoran al iniciar las tareas hasta que no sea factible completarlas, llevándolo a cuestionar sus habilidades y capacidades (Atalaya y García, 2019, Ayala et al., 2020).

Ahora bien, desde un punto de vista explicativo relevante de la dilación académica figura la asociación con la personalidad, en especial desde el modelo teórico de los cinco grandes factores (5GF). Este modelo propuesto por McCrae y Costa explica que los rasgos de la personalidad están compuestos por las tendencias básicas y las adaptaciones características. Lo primero (tendencias básicas) involucra lo genético que está relacionado con los rasgos de extraversión, neuroticismo, responsabilidad, apertura y amabilidad que son susceptibles a ser cambiadas en edades tempranas por distintas situaciones sociales; lo segundo (adaptaciones características) tiene que ver con la adaptación al medio, es decir es la expresión concreta de las tendencias básicas e involucra las habilidades, hábitos, actitudes, creencias y relaciones interpersonales que resultan de la interacción del individuo con su medio (Sánchez y Ledesma, 2007).

Hasta la actualidad se realizaron algunas investigaciones que relacionan las dimensiones de la personalidad y la dilación académica. Siendo así que la PA evidencia una relación inversa 
con el rasgo de responsabilidad y una relación directa con el factor neuroticismo (Guerra, 2018; Matrángolo, 2018). Por otro lado, una relación poco significativa con los rasgos de extraversión, amabilidad y apertura (Sánchez, 2017). Otro estudio realizado por Abood, Gazo, Alharbi y Mhaidat (2019), con una muestra de 385 estudiantes de la universidad de Hashemite Jordan, se observó una relación nula entre la PA con la extraversión y neuroticismo; además de una relación inversa con responsabilidad, y de menor grado con amabilidad y apertura.

Cabe recalcar que en el Perú existen pocos artículos que explican la relación entre ambas variables usando el inventario de los cinco grandes (BFI), asimismo en la ciudad de Tarapoto no se realizaron ningún estudio al respecto, ni mucho menos con una población universitaria con características cristianas, por lo que se vio oportuno realizar la presente investigación que tiene como objetivo determinar la relación entre las dimensiones de la personalidad y la procrastinación académica en una universidad peruana de la ciudad de Tarapoto, 2020.

\section{Metodología}

El presente estudio corresponde a un diseño no experimental transversal de tipo correlacional porque se buscó encontrar la relación entre los factores de la personalidad y la procrastinación académica. De diseño no experimental, puesto que, no se realizó ninguna manipulación de las variables, sino que se observó en contextos naturales (Hernández et al., 2014).

\section{Participantes}

Los participantes pertenecen a una Universidad Privada en la ciudad de Tarapoto que se encuentra ubicado en el centro Oeste del Perú en el Departamento de San Martín. El muestreo empleado fue no probabilístico por conveniencia debido a que la muestra de la población estuvo compuesta por estudiantes disponibles que proporcionaban mayor proximidad y accesibilidad para la investigación. Primeramente, se estableció contacto con los docentes y a través de ellos se encuestó a los estudiantes con un formulario en línea. Luego del cierre de la encuesta se tuvo la participación de 322 estudiantes, posteriormente se procedió a un control de calidad de las encuestas obtenidas excluyendo a las encuestas incompletas o mal realizadas, por lo que se consideró adecuado utilizar 240 casos, 81 (33.8\%) varones y 159 (66.3\%) mujeres. Los estudiantes pertenecían a las carreras profesionales de Psicología, Administración y Gestión Empresarial, Contabilidad y Gestión tributaria, donde se obtuvieron un total de $\mathrm{N}=240$ estudiantes, distribuida de la siguiente manera: 


\section{Tabla 1}

Distribución de los participantes según género y carrera profesional de una universidad privada

\begin{tabular}{|c|c|c|c|c|c|}
\hline \multirow{3}{*}{ Género } & \multicolumn{4}{|c|}{ Carrera Profesional } & \multirow{3}{*}{ Total (\%) } \\
\hline & \multirow[b]{2}{*}{ Psicología (\%) } & \multicolumn{2}{|c|}{ Administración } & Contabilidad & \\
\hline & & $\begin{array}{l}\text { Gestión } \\
\text { (\%) }\end{array}$ & Empresarial & $\begin{array}{l}\text { Gestión Tributaria } \\
\text { (\%) }\end{array}$ & \\
\hline Hombre & $43(30.1)$ & $21(41.2)$ & & $17(37.0)$ & $81(33.8)$ \\
\hline Mujer & 100 (69.9) & 30 (58.8) & & $29(63.0)$ & 159 (66.3) \\
\hline Total & 143 (59.6) & 51 (21.3) & & $46(19.2)$ & $240(100)$ \\
\hline
\end{tabular}

\section{Instrumentos}

a) La escala de procrastinación académica (EPA), Creado en Canadá por Deborah Ann Busko (1998), adaptada a una población peruana por Domínguez et al., (2014), quien reportó la validez del constructo a través de un análisis factorial exploratorio $(\mathrm{KMO}=.812$; esfericidad de Bartlett $\mathrm{p}<.01$; valor de Eigen=1.81 primer factor, valor de Eigen=4.13 segundo factor) confirmatorio (GFI= .97; RMSEA [90\%]=.078 [.066; .091]; RMR=.064), además de una adecuada consistencia interna que se estimó mediante el coeficiente de alfa y omega obteniéndose un $\alpha=.821$ (IC al 95\%: .793; .847), $\omega=.829$ para el factor autorregulación académica, y un $\alpha=.752$ (IC al 95\%: .705; .792), $\omega=.794$ para el factor postergación de actividades. Se trabajó con una muestra de 379 universitarios de la ciudad de Lima, cuyas edades oscilan entre 16 y 40 años. El cuestionario está conformado por 12 ítems tipo Likert de cinco opciones de respuesta, que van desde "nunca” hasta "siempre”, asimismo, es de administración individual o colectiva. Evalúa dos dimensiones, la postergación de actividades conformadas por (ítems 1, 6, 7) y autorregulación académica (ítems 2, 3, 4, 5, 8, 9, 10, 12).

b) El Big Five Inventory (BFI) versión utilizada para medir los cinco grandes factores de personalidad creado por McCrae, R. y Costa, P. (1985); adaptada y validada a una versión española con 44 ítems por Benet-Martínez y John (1998). Posteriormente Domínguez y Merino (2018) validaron el instrumento en el Perú mediante un análisis factorial confirmatorio (CFI= .939; RMSEA [90\%]= .073 [.065, .082]) y un modelamiento exploratorio de ecuación estructurales ESEM (CFI = 1.0; RMSEA [90\%] $=0.00[0.00, .029]$; ISF > .70) y una consistencia interna favorable estimada mediante el coeficiente de alfa y omega en las dimensiones de neuroticismo $(\alpha=.559, \omega=.669$; ítems $2,6,10)$, responsabilidad $(\alpha=.611$, 
$\omega=.608$; ítems 5,8,11), amabilidad $(\alpha=.767, \omega=.683$; ítems $3,12,14)$, extraversión $(\alpha=.757$, $\omega=.705$; ítems $1,4,15)$ y apertura $(\alpha=.567, \omega=.627$; ítems $2,6,10)$. Se trabajó con una muestra de 534 universitarios de la ciudad de Lima, cuyas edades comprenden de 16 a 38 años. El inventario está compuesto de 15 ítems tipo Likert de cinco opciones en respuesta, que van desde “muy en desacuerdo” hasta “muy de acuerdo”, de administración individual o colectiva.

\section{Procedimiento y ética}

En primer lugar, se solicitó una autorización a los coordinadores de las Facultades de Ciencias de la Salud y Ciencias Empresariales para encuestar a sus universitarios. Posteriormente se instó a los docentes un espacio en su horario de clases para presentar el proyecto de investigación a los estudiantes y solicitar la participación de los mismos, consecuentemente se les envió un enlace de google formularios (https://forms.gle/1ECgiLwmr3oXbeer7) a la plataforma del chat de la clase virtual. Cabe recalcar que los participantes fueron informados que su participación era voluntaria y anónima. Asimismo, se les explicó los propósitos de la investigación, declarando su consentimiento en la primera parte del cuestionario en línea.

El primer criterio de inclusión fue que el estudiante este matriculado en la facultad de Ciencias de la Salud y Ciencias Empresariales durante el año 2020-1, el segundo criterio fue la participación de ambos sexos y el tercer criterio fue la participación de estudiantes en edades comprendidas entre los 16 a 40 años.

Luego de la recogida de datos, se procedió a examinar y descartar encuestas incompletas, preparándolos para el tratamiento en SPSS (V.26) en español. El proceso estadístico comprendió un primer análisis descriptivo y de supuestos de normalidad a través de la prueba de Kolgomorov Smirnov. Finalmente se utilizó la prueba estadística Spearman para determinar la correlación entre las dimensiones de la personalidad y la procrastinación académica, además de describir los niveles de procrastinación según edad y sexo en estudiantes de cursos online de una universidad peruana de la ciudad de Tarapoto, 2020.

\section{Resultados}

\section{Análisis descriptivos}

Con respecto a la procrastinación académica en la tabla 2, se observa una mayor frecuencia del nivel medio en edades de 16 a 19 años (48.1\%) y de 20 a 24 años (41.0\%), un 
nivel alto en edades entre 25 a 40 años (45.2\%). Sobre la variable sexo se puede apreciar en ambos una mayor frecuencia del nivel medio en hombres (44.4\%) y en mujeres (42.8\%).

\section{Tabla 2}

Niveles de procrastinación académica por características demográficas

\begin{tabular}{llll}
\hline Características & Bajo (\%) & Medio (\%) & Alto (\%) \\
\hline Edad & & & \\
$16-19$ & $35(33.7)$ & $50(48.1)$ & $19(18.3)$ \\
$20-24$ & $35(33.3)$ & $43(41.0)$ & $27(25.7)$ \\
$25-40$ & $6(19.4)$ & $11(35.5)$ & $14(45.2)$ \\
Sexo & & & \\
Hombre & $22(27.2)$ & $36(44.4)$ & $23(28.4)$ \\
Mujer & $54(34.0)$ & $68(42.8)$ & $37(23.3)$ \\
Total & $76(31.7)$ & $104(43.3)$ & $60(25.0)$ \\
\hline
\end{tabular}

En lo referente a postergación de actividades la tabla 3, se evidencia un mayor porcentaje de nivel bajo en edades comprendidas entre 16 a 19 años (48.1\%), 20 a 24 años (37.1\%) y 25 a 40 años (48.4\%). En cuanto a la variable sexo encontramos una mayor distribución de nivel medio en hombres (45.7\%) y un nivel bajo en mujeres (49.7\%).

Tabla 3

Niveles de postergación de actividades por características demográficas

\begin{tabular}{llll}
\hline Características & Bajo (\%) & Medio (\%) & Alto (\%) \\
\hline Edad & $50(48.1)$ & $42(40.4)$ & $12(11.5)$ \\
$16-19$ & $39(37.1)$ & $39(37.1)$ & $27(25.7)$ \\
$20-24$ & $15(48.4)$ & $10(32.3)$ & $6(19.4)$ \\
$25-40$ & & & \\
Sexo & & $37(45.7)$ & $19(23.5)$ \\
Hombre & $5(30.9)$ & $54(34.0)$ & $26(16.4)$ \\
Mujer & $79(49.7)$ & $91(37.9)$ & $45(18.8)$ \\
Total & $104(43.3)$ & & \\
\hline
\end{tabular}


En lo concierne a autorregulación académica, la tabla 4 muestra una mayor frecuencia del nivel medio en edades de 16 a 19 años (44.2\%) y de 20 a 24 años (43.8\%), un nivel bajo en edades de 25 a 40 años (51.6\%). Con respecto a la variable sexo se observa una mayor frecuencia de nivel medio en mujeres (44.0\%), mientras que en hombres existe una pequeña diferencia de $1.2 \%$ entre el nivel medio (37.0\%) y alto (35.8\%).

Tabla 4

Niveles de autorregulación académica por características demográficas

\begin{tabular}{llll}
\hline Características & Alto (\%) & Medio (\%) & Bajo (\%) \\
\hline Edad & & & \\
$16-19$ & $41(39.4)$ & $46(44.2)$ & $17(16.3)$ \\
$20-24$ & $36(34.3)$ & $46(43.8)$ & $23(21.9)$ \\
$25-40$ & $7(22.6)$ & $8(25.8)$ & $16(51.6)$ \\
Sexo & & & \\
Hombre & $29(35.8)$ & $30(37.0)$ & $22(27.2)$ \\
Mujer & $55(34.6)$ & $70(44.0)$ & $34(21.4)$ \\
Total & $84(35.0)$ & $100(41.7)$ & $56(23.3)$ \\
\hline
\end{tabular}

Con el objetivo de realizar las correlaciones en las variables de estudio y contrastar las hipótesis. Se procedió a aplicar la prueba de normalidad de Kolmogorov Smirnov que se presenta en la tabla 5, el cual registra una distribución asimétrica, puesto que presenta en todas sus dimensiones una significancia menor a 0.05 , por lo que se opta a la prueba no paramétrica para contrastar las hipótesis, en este caso el coeficiente de correlación "rho” de Spearman.

\section{Tabla 4}

Prueba de normalidad de Kolmogorov - Smirnov de las variables de estudio

\begin{tabular}{|c|c|c|c|c|c|c|}
\hline Variable & Dimensión & Media & D.E. & k-s & gl & $\mathrm{p}$ \\
\hline \multirow{3}{*}{$\begin{array}{l}\text { Procrastinación } \\
\text { académica }\end{array}$} & $\begin{array}{l}\text { Postergación de } \\
\text { actividades }\end{array}$ & 7.27 & 2.538 & 0.125 & 240 & 0.003 \\
\hline & $\begin{array}{l}\text { Autorregulación } \\
\text { académica }\end{array}$ & 17.6 & 5.988 & 0.111 & 240 & 0.000 \\
\hline & $\begin{array}{l}\text { Procrastinación } \\
\text { académica }\end{array}$ & 24.87 & 7.115 & 0.74 & 240 & 0.000 \\
\hline
\end{tabular}




\begin{tabular}{lllllll} 
& Neuroticismo & 8.5 & 2.837 & 0.072 & 240 & 0.004 \\
\multirow{3}{*}{ Personalidad } & Responsabilidad & 12.79 & 2.127 & 0.177 & 240 & 0.000 \\
& Amabilidad & 13.27 & 2.063 & 0.200 & 240 & 0.000 \\
& Extraversión & 11.39 & 2.5 & 0.129 & 240 & 0.000 \\
& Apertura & 12.28 & 2.121 & 0.141 & 240 & 0.000 \\
\hline
\end{tabular}

La tabla 6, se observa que existe una correlación inversa significativa entre las dimensiones de la personalidad y la procrastinación académica; responsabilidad (rho= -0.590 ; $\mathrm{p}<.001$ ), amabilidad (rho $=-0.457 ; \mathrm{p}<.000$ ), extraversión (rho $=-0.226 ; \mathrm{p}<.000$ ) y apertura $(\mathrm{rho}=-355 ; \mathrm{p}<.000)$; quiere decir que a mayor responsabilidad, amabilidad, extraversión y apertura menor será la procrastinación académica o viceversa. Caso contrario sucede con la dimensión neuroticismo que presenta una significativa correlación directa (rho=0.166; $\mathrm{p}<$ .000), de modo que a mayor neuroticismo, mayor procrastinación académica.

\section{Tabla 5}

Correlación de Spearman de procrastinación académica y las dimensiones de la personalidad

\begin{tabular}{llll}
\hline & N & rho de Spearman & p \\
\hline Procrastinación Académica - Neuroticismo & 240 & 0.166 & 0.001 \\
Procrastinación Académica - Responsabilidad & 240 & -0.590 & 0.000 \\
Procrastinación Académica - Amabilidad & 240 & -0.457 & 0.000 \\
Procrastinación Académica - Extraversión & 240 & -0.226 & 0.000 \\
Procrastinación Académica - Apertura & 240 & -0.355 & 0.000 \\
\hline
\end{tabular}

\section{Discusión}

El objetivo de la presente investigación es analizar la relación entre las dimensiones de la personalidad y la procrastinación académica en los estudiantes que pertenecen a las Facultades de Ciencias de la Salud y Ciencias Empresariales de una universidad peruana de la ciudad de Tarapoto, Perú. Los resultados encontrados indican que existe una débil correlación directa y significativa entre la dimensión neuroticismo y procrastinación académica $(r h o=.166$; $\mathrm{p}<$.001). Datos que coinciden con los de Lee et, al. (2006) quienes confirman que un neuroticismo elevado predice la procrastinación crónica de las decisiones. De la misma manera Sánchez (2017) evidencia que estudiantes con mayor neuroticismo presentan dificultad para 
poder realizar sus deberes académicos y plantearse metas dentro del mismo. Cabe recalcar que las razones por las cuales este tipo de personas procrastinan es por el miedo al fracaso, ansiedad por el rendimiento, perfeccionismo y falta de confianza (Maldonado y Zenteno, 2018), conllevándolos (as) a pensar que todo les saldrá mal y exagerando las consecuencias de una acción (Tibbett y Ferrari, 2015).

Contrariamente se observa una moderada correlación inversa y significativa (rho= .590; $\mathrm{p}$ < .000) entre la dimensión responsabilidad y procrastinación académica, resultado que guarda relación con otros estudios (Corkin et al., 2011; Kuhnle et al., 2011) que demuestran que la procrastinación se relaciona negativamente con la autorregulación adaptativa. Esto se debe a que el factor responsabilidad está asociado con el sentido del deber, autodisciplina, competencia, reflexión y el cumplimiento de normas (Sánchez y Ledesma, 2007) que ocasiona a una mayor autorregulación, por consecuencia una menor dilación (Domínguez et al., 2019). Asimismo, los estudiantes concienzudos pueden manejar mejor su comportamiento para cumplir con los plazos establecidos y alcanzar sus objetivos (Zhou, 2019). Otro estudio demuestra que los alumnos procrastinan menos cuando manifiestan la intención decidida de acabar con la tarea y eso se debe a un nivel alto de conciencia como rasgo de personalidad (Ayala et.al., 2020).

Sobre la relación entre la dimensión amabilidad y la procrastinación académica se obtuvo una moderada correlación inversa y significativa (rho $=-.457 ; \mathrm{p}<.000$ ), resultado que es congruente con algunos estudios (Abood et. al., 2019; Domínguez et. al., 2019, Kim et. al., 2017) y opuesto a otros, que sostienen que la amabilidad se caracteriza por la complacencia, distraibilidad y baja planificación que eventualmente interfiere con el cumplimiento de tareas (Seddigh et al., 2016). El factor amabilidad indica calidad en las interacciones sociales y se caracteriza por la sensibilidad, confianza, altruismo y franqueza que ocasiona gratitud y felicidad en la persona (Alarcón y Morales de Isasi, 2012), y en consecuencia una mayor eficacia en los trabajos académicos (Garcia, 2015; Ramirez y Fuentes, 2013). Por otro lado, también puede deberse a que este tipo de estudiantes sean responsables con sus tareas porque piensan que otros dependerán de su trabajo o apoyo (Kim et al., 2017). Probablemente nuestro resultado se vea influido por la religiosidad, principios y valores cristianos que promueve la Universidad y como es sabido la religiosidad está asociada al factor amabilidad (Koenig et al., 2012).

Acerca de la dimensión extraversión y su relación con la procrastinación académica se obtuvo una débil correlación inversa pero significativa (rho= -.226; $\mathrm{p}<.000$ ), estos hallazgos 
son concordantes con Abood et al., (2019) que encontró una correlación inversa entre extraversión y procrastinación académica, asimismo, Watson (2000) demuestra una correlación significativa entre procrastinación de actividades generales con una baja extraversión. Es posible que los extrovertidos sean menos tendientes a la postergación debido a que son sujetos involucrados y asertivos que les gustan hacer varias tareas a un ritmo acelerado y tomar control sobre la situación (Kim et al., 2017). Los resultados obtenidos concuerdan con la investigación de Bastidas (2017) quien aduce que este tipo de personas tienen una mayor capacidad de lograr una mejor comprensión de los deseos y metas personales, llegando a planificar su tiempo de forma eficaz.

En cuanto a la dimensión apertura y su relación con la procrastinación académica se encontró una débil correlación inversa pero significativa (rho= -.355; $\mathrm{p}<.000$ ), en este sentido Zhou (2019) encuentra relación directa entre apertura y la capacidad de cumplir con los plazos y objetivos. El factor apertura a la experiencia distingue a personas creativas, con intereses intelectuales y artísticos, imaginativos y sensibles a la belleza, con mayor conciencia de sus sentimientos y un pensamiento concreto (Matrángolo, 2018), características que ayudan a crear buenas relaciones con los profesores y compañeros, integrándose al entorno universitario, favoreciéndoles en su autoeficacia y adaptación académica (Abood et al., 2020). Estos resultados probablemente se deban a que los estudiantes con estas características tengan un alto grado de curiosidad intelectual y ganas de aprender, así como la capacidad de cumplir con los plazos y objetivos (Komarraju et al., 2011).

En conclusión, la procrastinación académica guarda relación con las dimensiones de la personalidad. Siendo así que los factores de responsabilidad y amabilidad presentan una moderada relación inversa, por lo que se puede concluir que, a mayor predominio de estos rasgos, menor será la procrastinación académica o viceversa; asimismo, las dimensiones apertura y extraversión evidencia una baja correlación inversa y significativa, por lo que se puede concluir que, a mayor desenvolvimiento de estos rasgos, disminuirá la dilación académica o viceversa. Contrariamente el factor neuroticismo ya que señala una débil correlación directa y significativa, pudiéndose inferir que a mayor neuroticismo, mayor procrastinación académica.

Considerando las implicaciones prácticas, el presente estudio tiene gran importancia en el Perú, ya que existen pocos artículos que relacionan ambas variables. Este hallazgo evidencia que para disminuir la procrastinación es recomendable aumentar las características asociadas a las dimensiones de responsabilidad, amabilidad, extraversión y apertura; en especial a los dos 
primeros debido a que su relación con la procrastinación la moderada. Considerándose que la responsabilidad está asociada a la realización de objetivos seguidos de una buena planificación de tareas y organización del tiempo e involucra emplear esfuerzos razonables necesarios para completar la tarea. Por otro lado, la dimensión amabilidad está asociado con actitud conciliadora, de sensibilidad social, altruista y cooperativa, por lo que desarrollar estas características facilitaría la paz mental y la felicidad lo que ayudará en el desempeño académico, como los resultados de esta investigación demuestran.

Con respecto a las limitaciones del estudio, si bien este estudio proporciona información relevante sobre la relación entre las dimensiones de la personalidad y la procrastinación académica, no se debe generalizar los resultados ya que la muestra es limitada, además hubo una mayor participación de mujeres. Otra limitación o desventaja es que la encuesta se desarrolló virtualmente, por lo que, si hubiera sido presencialmente, se habría concedido una mayor interacción con los estudiantes y así aumentar su compromiso en su participación, además de observar su actitud en el desarrollo de la encuesta.

\section{Conclusiones}

La investigación contó con dos fortalezas, la primera es que se realizó de manera virtual, lo que facilitó un mayor alcance de participantes y recoger información de manera rápida; la segunda fortaleza se debe al recojo de encuestas se realizó en un momento en el cual los estudiantes fueron completamente responsables de su tiempo dentro y fuera de clases debido a que las mismas eran virtuales, además de ser consciente de la coyuntura actual de la crisis sanitaria mundial; siendo esto un factor influyente a desarrollar nuevas conductas para afrontar su nueva realidad (Organización Mundial de la Salud [OMS], 2020).

Finalmente, se recomienda para futuras investigaciones detallar la influencia del género con los factores de la personalidad y su relación con la procrastinación en estudiantes de educación medio, superior y tecnología de carácter público y privada, asimismo considerar la edad en la conducta procrastinante ya que esta aumenta con el pasar de los años (Domínguez et. al., 2019).

\section{Referencias}

Abood M., Alharbi B., Mhaidat F., y Gazo A. (2020). The Relationship between Personality Traits, Academic Self-Efficacy and Academic Adaptation among University Students in Jordan. International Journal of Higher Education, 9(3), 120-128. https://doi.org/10.5430/ijhe.v9n3p120 
Abood M., Gazo A., Alharbi B. y Mhaidat F. (2019). The Relationship between Academic Procrastination and Personality Traits According to the Big Five Personality Factors Model among Students of University. Dirasat Educational Sciences, 46(1), 784-794. https://dirasat.ju.edu.jo/ES/Article/ViewArticle?volume=46\&issue=1\&articleId=1362 9

Alarcón, R. y Morales de Isasi, C. (2012). Relaciones entre gratitud y variables de personalidad. Acta de investigación psicológica 2(2), 699-712. https://www.researchgate.net/publication/262633755_Relaciones_entre_Gratitud_y_V ariables_de_Personalidad

Atayala, C. y García, L. (2019). Procrastinación: Revisión teórica. Revista de Investigación en Psicología, 22(2), 363-378. http://dx.doi.org/10.15381/rinvp.v22i2.17435

Ayala, A., Rodríguez, R., Villanueva, W., Hernández, M., y Campos, M. (2020). Procrastinación académica: teorías, elementos y modelos. Muro de la Investigación, 5(2). https://doi.org/10.17162/rmi.v5i2.1324

Bastidas, J. (2017). Procrastinación y rasgos de personalidad en estudiantes de una Universidad Privada de Lima este, 2016 [Tesis de Licenciatura, Universidad Peruana Unión]. Repositorio Institucional - Universidad Peruana Unión. http://repositorio.upeu.edu.pe/handle/UPEU/411

Benet-Martinez, V. y John, O. (1998). Across Cultures and Ethnic Groups: Multitrait Multimethod Analyses of the Big Five in Spanish and English. Journal of Personality and Social Psychology, 75(3), 729-750. https://doi.org/10.1037/0022-3514.75.3.729

Castro S. y Mahamud, K. (2017). Procrastinación académica y adicción a internet en estudiantes universitarios de Lima Metropolitana. Avances en Psicología Latinoamericana, 25(2), 189-197. https://doi.org/10.33539/avpsicol.2017.v25n2.354

Cerqueira, C., Castro, M., Beltrán, P. y Tello, C. (2016). Relación entre procrastinación general $Y$ académica y dimensiones de personalidad en estudiantes universitarios [Tesis de Licenciatura, Universidad San Pedro]. Repositorio Institucional - Universidad San Pedro. http://repositorio.usanpedro.edu.pe/handle/USANPEDRO/69

Chen, Z., Zhang, R., Xu, T., Yang, Y., Wang, J. y Feng, T. (2020). Emotional attitudes towards procrastination in people: A large-scale sentiment-focused crawling analysis. Computers in Human Behavior, 110(2), 106391. https://doi.org/10.1016/j.chb.2020.106391

Contreras, H., Mori, E., Lam, N., Gil, E., Hinostrosa, W., Rojas, D., Espinoza, E., Torrejón, E. y Conspira, C. (2011). Procrastinación en el estudio: exploración del fenómeno en adolescentes escolarizados. Lima Metropolitana, Perú. Revista Peruana de Epidemiología, 15(3), 1-5. http://www.redalyc.org/articulo.oa?id=203122771007

Corkin, D., Yu, S. y Lindt, S. (2011). Comparing active delay and procrastination from a selfregulated learning perspective. Learning and Individual Differences, 21(5), 602-606. https://doi.org/10.1016/j.lindif.2011.07.005

Díaz-Morales, J. (2018). Procrastinación: Una revisión de su medida y sus correlatos. Revista 
Iberoamericano de Diagnóstico y Evaluación, 2(51), 43-60.

https://www.aidep.org/sites/default/files/2019-04/RIDEP51-Art4.pdf

Domínguez, S., Villegas, G. y Centeno, S. (2014). procrastinación académica: validación de una escala de una muestra de estudiantes de una universidad privada. Liberabit, 20(2), 293-304. http://www.scielo.org.pe/pdf/liber/v20n2/a10v20n2.pdf

Domínguez, S. y Merino, C. (2018). Dos versiones breves del Big Five Inventory en universitarios peruanos: BFI-15p y BFI-10p. Revista Peruana de Psicología, 24(1), 8196. https://doi.org/10.24265/liberabit.2018.v24n1.06

Domínguez, S., Prada, R. y Moreta, R. (2019). Diferencias de género en la influencia de la personalidad sobre la procrastinación académica en estudiantes universitarios peruanos.

Acta Colombiana de Psicología, 242(2), 125-136. http://www.doi.org/10.14718/ACP.2019.22.2.7

Ferreyra, M., Avitabile, C., Álvarez, J., Haimovich, Francisco y Urzúa, S. (2017). Momento decisivo: La educación superior en América Latina y el Caribe. In Banco Mundial. https://doi.org/10.1103/PhysRevB.33.1471

García, J. (2015). Gratitud en el Aula. Reidocrea, 4, 6-13. https://digibug.ugr.es/bitstream/handle/10481/34596/ReiDoCrea-Vol.4-Art.1Garcia.pdf

Kim, S., Fernández, S. y Terrier, L. (2017). Procrastination, personality traits, and academic performance: When active and passive procrastination tell a different story. Personality and Individual Differences, 108, 154-157. https://doi.org/10.1016/j.paid.2016.12.021

Koenig, H., King, D. y Carson, V. (2012). Handbook of religion and health. In Oxford University Press, vol. 2. https://doi.org/10.1136/jech.55.9.688

Komarraju, M., Karau, S. J., Schmeck, R. R. y Avdic, A. (2011). The Big Five personality traits, learning styles, and academic achievement. Personality and Individual Differences, 51(4), 472-477. https://doi.org/10.1016/j.paid.2011.04.019

Kuhnle, C., Hofer, M. y Kilian, B. (2011). The Relationship of Self-control, Procrastination, Motivational Interference and Regret with School Grades and Life Balance. Journal of Childhood and Adolescence Research, 6(1), 31-44. https://nbnresolving.org/urn:nbn:de:0168-ssoar-385735

Laureano, C. y Ampudia, L. G. (2019). Procrastinación: Revisión Teórica. Revista de Investigación en Psicología, 22, 363-378. https://doi.org/10.15381/rinvp.v22i2.17435

Lee D., Kelly K. y Edwards J. (2006). A closer look at the relationships among trait procrastination, neuroticism, and conscientiousness. Personality and Individual Differences 40 (1), 27-37. https://doi.org/10.1016/j.paid.2005.05.010

Matalinares, C., Diaz, A., Rivas, D., Dioses, C., Arenas, I., Villalba, R., Baca, D., Uceda, E., Fernández, E. y Yaringaño, L. (2017). Procrastinación y adicción a redes sociales en estudiantes universitarios de pre y postgrado de Lima. Horizonte de La Ciencia, 7(13), 63-81. http://revistas.uncp.edu.pe/index.php/horizontedelaciencia/article/view/355 
Matrángolo, G. (2018). Dejar para mañana. personalidad y procrastinación académica. Hologramática, 27(32), 3-10. https://www.cienciared.com.ar/ra/doc.php?n=2077

McCrae, R. y Costa, P. (1985). Comparison of EPI and psychoticism scales with measures of the five-factor theory of personality. Personality and individual Differences, 6, 587-597. https://doi.org/10.1016/0191-8869(85)90008-X

Organización Mundial de la Salud. (14 de abril de 2020). Actualización de la estrategia frente al Covid-19. https://www.who.int/docs/default-source/coronaviruse/covid-strategyupdate-14april2020

Quiroga, L. (2020). Procrastinación y estilos de crianza en estudiantes de una universidad de Chiclayo [Tesis de Maestro, Universidad Cesar Vallejo]. Repositorio Institucional Universidad Cesar Vallejo. https://tesis.ucsm.edu.pe/repositorio/handle/UCSM/3518

Ramírez, P. y Fuentes, C. (2013). Felicidad y rendimiento académico: Efecto moderador de la felicidad sobre los indicadores de selección y rendimiento académico de alumnos de Ingeniería Comercial. Formación Universitaria, 6(3), 21-30. http://dx.doi.org/10.4067/S0718-50062013000300004

Sánchez, C. (2017). Personalidad y procrastinación académica en estudiantes de la facultad de medicina humana y ciencias de la salud de la universidad Alas Peruanas, Lima 2017 [Tesis de Licenciatura, Universidad Alas Peruanas] Repositorio Institucional Universidad Alas Peruanas. http://repositorio.uap.edu.pe/handle/uap/8183

Sánchez, R. y Ledesma, R. (2007). Los Cinco Grandes Factores: cómo entender la personalidad y cómo evaluarla. Serie Investigación y Desarrollo, 131-160. https://www.researchgate.net/publication/338111357

Steel P. y Ferrari J. (2013). Sex, Education and Procrastination: An Epidemiological Study of Procrastinators' Characteristics from a Global Sample. European Journal of Personality, 27(1), 51-58. https://doi.org/10.1002/per.1851

Hernández, R., Fernández, C., \& Baptista, M. (2014). Metodología de la Investigación. Santa Fe: Mc Graw Hill.

Seddigh A., Berntson E., Platts L. y Westerlund H. (2016). Does Personality Have a Different Impact on Self-Rated Distraction, Job Satisfaction, and Job Performance in Different Office Types?. PLoS ONE 11(5): e0155295. https://doi.org/10.1371/journal.pone.0155295

Tibbett, T. y Ferrari, J. (2015). The portrait of the procrastinator: Risk factors and results of an indecisive personality. Personality and Individual Differences, 82, 175-184. https://doi.org/10.1016/j.paid.2015.03.014

Valverde C. (2020). Estilos de crianza y procrastinación académica en estudiantes adolescentes Lima Norte, 2020 [Tesis de Licenciatura, Universidad Cesar Vallejo]. Repositorio Institucional - $\quad$ Universidad Cesar Vallejo. https://hdl.handle.net/20.500.12692/47827 
Watson, D. (2000). Procrastination and the five-factor model: a facet level analysis. Personality and Individual Differences, 30 (2001), 149-158. https://www.elsevier.com/locate/paid

Zhou, M. (2019). The role of personality traits and need for cognition in active procrastination. Acta Psychologica, 199, 102883. https://doi.org/10.1016/j.actpsy.2019.102883 